\title{
Genome-wide identification and expression analysis of the trehalose-6-phosphate synthase (TPS) gene family in cucumber (Cucumis sativus L.)
}

\author{
Yuanyuan Dan ${ }^{1}$, Yuan Niu $^{1}$, Chunlei Wang ${ }^{1}$, Mei Yan $^{1}$, Weibiao Liao ${ }^{\text {Corresp. } 1}$ \\ ${ }^{1}$ Gansu Agricultural University, Lanzhou, China \\ Corresponding Author: Weibiao Liao \\ Email address: liaowb@gsau.edu.cn
}

Trehalose-6-phosphate synthase (TPS) is significant in the growth, development, and stress resistance of plants. We identified the cucumber TPS family and its physicochemical properties, domains, gene structures, evolutionary relationships, gene locations, cis-acting elements, conserved motifs, and expression patterns using bioinformatics. Our results uncovered seven CSTPS genes in the cucumber genome and named CSTPS1-CSTPS7 according to their locations in the chromosomes. Seven CSTPS genes were randomly distributed in six cucumber chromosomes. Domain analysis showed that the TPS and TPP domains exist in all CSTPSs, and an additional hydrolase-3 domain exist in CSTPS3, CSTPS5, and CSTPS6. Phylogenetic analysis showed that TPS proteins from Arabidopsis, rice, soybean, and cucumber were divided into two subfamilies (Class I and Class II) and they were further divided into seven subgroups. TPS proteins from Arabidopsis and cucumber were grouped together, suggesting a close evolutionary relationship. Gene structure analysis indicated that most Class I genes contained 16-17 introns, while Class II genes (except CSTPS7) had two introns. Motif analysis showed that Class II genes had 10 complete conserved motifs, while Class I genes lacked motif 8 and motif 9. Furthermore, CSTPS genes possessed numerous cis-acting elements related to stress, hormone, and light response in the promoter regions. $\mathrm{GO}$ analysis indicated multiple functions for the CSTPS proteins. Expression analysis of CSTPS genes in different tissues found that they were expressed in roots, stems, and leaves, with the highest expression levels in roots. The expression analysis of CSTPSs under different treatments showed that CSTPS genes may participate in the response to abiotic stress, plant hormones, and sugar treatments. 


\section{Genome-wide identification and expression analysis of the}

2 trehalose-6-phosphate synthase (TPS) gene family in

3 cucumber (Cucumis sativus $\mathbf{L}$.)

4

5

6 Yuanyuan Dan ${ }^{1}$, Yuan Niu ${ }^{1}$, Chunlei Wang ${ }^{1}$, Mei Yan ${ }^{1}$, Weibiao Liao ${ }^{1}$

7

$8 \quad{ }^{1}$ Gansu Agricultural University, Lanzhou, China

9

10 Corresponding Author: Weibiao Liao

11 Gansu Agricultural University, Lanzhou, China

12 Email address: liaowb@gsau.edu.cn 
14

15

16

17

18

19

20

21

22

23

24

25

26

27

28

29

30

31

32

33

34

35

36

37

38

39

40

41

42

43

44

45

46

47

48

49

50

51

52

\section{Abstract}

Trehalose-6-phosphate synthase (TPS) is significant in the growth, development, and stress resistance of plants. We identified the cucumber TPS family and its physicochemical properties, domains, gene structures, evolutionary relationships, gene locations, cis-acting elements, conserved motifs, and expression patterns using bioinformatics. Our results uncovered seven CsTPS genes in the cucumber genome and named CsTPS1-CsTPS7 according to their locations in the chromosomes. Seven CSTPS genes were randomly distributed in six cucumber chromosomes. Domain analysis showed that the TPS and TPP domains exist in all CsTPSs, and an additional hydrolase-3 domain exist in CsTPS3, CsTPS5, and CsTPS6. Phylogenetic analysis showed that TPS proteins from Arabidopsis, rice, soybean, and cucumber were divided into two subfamilies (Class I and Class II ) and they were further divided into seven subgroups. TPS proteins from Arabidopsis and cucumber were grouped together, suggesting a close evolutionary relationship. Gene structure analysis indicated that most Class I genes contained 16-17 introns, while Class II genes (except CsTPS7) had two introns. Motif analysis showed that Class II genes had 10 complete conserved motifs, while Class I genes lacked motif 8 and motif 9. Furthermore, CSTPS genes possessed numerous cis-acting elements related to stress, hormone, and light response in the promoter regions. GO analysis indicated multiple functions for the CsTPS proteins. Expression analysis of CsTPS genes in different tissues found that they were expressed in roots, stems, and leaves, with the highest expression levels in roots. The expression analysis of CsTPSs under different treatments showed that CsTPS genes may participate in the response to abiotic stress, plant hormones, and sugar treatments.

\section{Introduction}

The growth and development of plants in agriculture are often affected by various adverse conditions, including submergence, drought, low or high temperatures, and saline and alkaline soils (Zhu, 2016). These adverse conditions may dehydrate plants, which reduces the photosynthesis rate (Baninasab, 2010), promotes the production of reactive oxygen species (ROS), and damages the cell membrane (Deinlein et al., 2014). Plants have produced a series of physiological mechanisms over time to protect themselves from adversity. For example, under stress conditions, plants express stress-related genes to produce stress proteins, including the heat shock protein (HSP), low temperature-induced protein, osmoregulatory protein, and the pathogenesis-related protein (PR) (Hightower, 1991). Plants may also accumulate osmotic adjustment substances (Ashraf et al., 2007) under unfavorable conditions, including inorganic ions $\left(\mathrm{Na}^{+}, \mathrm{K}^{+}\right.$, and $\mathrm{Cl}^{-}$), proline, betaine, abscisic acid (Kim, 2012), and sugars (sucrose, fructose, and trehalose) to protect the integrity of the membrane structure.

Trehalose ( $\alpha$-D-glucopyranosyl-1, 1- $\alpha$-D-glucopyranoside) (Lunn et al., 2014) is a non-reducing disaccharide composed of two molecules of glucose (Elbein et al., 2003). In higher plants, trehalose is synthesized through the catalysis of two enzymes: trehalose-6-phosphate synthase 
53 (TPS) and trehalose-6-phosphate phosphatase (TPP). TPS first catalyzes UDP-glucose and

54

55

56

57

58

59

60

61

62

63

64

65

66

67

68

69

70

71

72

73

74

75

76

77

78

79

80

81

82

83

84

85

86

87

88

89

90

91

92

glucose-6-phosphate to produce trehalose-6-phosphate (T6P) and UDP. Then, TPP

dephosphorylates trehalose-6-phosphate to produce trehalose (Goddijn et al., 1999). Trehalose is widely found in plants and plays a specific role in plant growth, development, and resistance to stress. Compost treated with trehalose has been used to cultivate quinoa plants to increase their growth and yield (Abdallah et al., 2020), and soaking rice seeds with trehalose may relieve salt stress (Abdallah et al., 2016). Trehalose may form Cd-Trehalose chelate with cadmium (Cd) to alleviate the damage of cadmium stress in rice (Wang et al., 2020). The expression of the TPS gene and the accumulation of trehalose has been shown to increase when wheat are exposed to drought (El-bashiti et al., 2005).

TPS plays a vital role in trehalose metabolism and stress resistance in plants (Yang et al., 2012). Previous studies have shown that light quality may affect the growth and phase transition by influencing the TPS1-T6P signaling pathway in tomatoes (Chen et al., 2017). The

overexpression of the TPS1 gene in rice and potato enhanced their stress resistance (Li et al., 2011, Kondrák et al., 2011). The overexpression of the TPS11 gene in wheat improved cold resistance in Arabidopsis (Liu et al., 2019); TPS1 played an important role in the embryogenesis, post-embryonic growth, and development in Arabidopsis (Fichtner et al., 2020). Studies have shown that TPS affected development and metabolic processes by altering T6P levels (Lunn et al., 2015)

T6P is an intermediary in trehalose biosynthesis with a vital role in plant growth and development (Yadav et al., 2014; Zhang et al., 2015). T6P serves as a sugar-signaling molecule in Arabidopsis that coordinates the hypocotyl elongation mediated by high temperature and the availability of endogenous sugar (Geonhee et al., 2019). The accumulation of T6P inhibited the growth of Arabidopsis seedlings mediated by trehalose (Schluepmann et al., 2004). The T6P signaling pathway played an important role in the flowering of the Arabidopsis leaf and stem meristems (Wahl et al., 2013).

T6P was also involved in regulating the use and distribution of sucrose, coordinating source-sink relationships, the efficient use of carbohydrates (Schluepmann et al., 2003), and improving crop yield (Paul et al., 2020). Research on cucumber fruit has shown that there was a strong correlation between T6P and sucrose (Zhang et al., 2015). The Tre6P:sucrose ratio could maintain sucrose levels within a range that is appropriate for the cell type and developmental stage of plants (Yadav et al., 2014). Varying T6P levels and sugar signaling through chemical intervention significantly impacted crop yield and resilience (Griffiths et al., 2016; Smeekens, 2017).

The overexpression of rice TPP genes in maize ears under well-watered or drought conditions reduced the level of T6P, increased the level of sucrose, and improved yield (Nuccio et al., 2015). A study of Arabidopsis indicated that many growth and developmental defects were due to T6P rather than trehalose (Schluepmann et al., 2003).

PeerJ reviewing PDF | (2020:12:56113:2:0:NEW 10 Apr 2021) 
93 Cucumber (Cucumis sativus L.) is a widely cultivated crop with high nutritional value. Its growth 94 and development are easily affected by adverse condition, especially salt stress (Miao et al., 95 2020). We sought to identify members of the TPS family in cucumber using bioinformatics 96 methods. We analyzed the gene structure and location, motif distribution and composition, 97 evolutionary relationship, and expression patterns. We hope that our work supports future 98 functional research of the TPS family in cucumber plants.

99

100

101

102

103

104

105

106

107

108

109

110

111

112

113

114

\section{Materials and Methods}

\section{Genome-wide identification and bioinformatics analysis}

We downloaded the whole genome in the gff, cds, pep, and fasta file format from the EnsemblPlants- Cucumber genome database (ASM407v2) (http://plants.ensembl.org/index.html) (Li et al., 2019). The TPS (Glyco-transf-20, PF00982) and TPP (Trehalose_PPase, PF02358) domains' hidden Markov Models (HMM) were downloaded from the Pfam database (http://pfam.xfam.org) (Liu et al., 2019). HMMsearch software and the TPS and TPP's HMMs were used to search all possible TPS candidate sequences containing typical TPS and TPP domains under a Linux system (Chen et al., 2019). Pfam (Liu et al., 2019) and NCBI-CDD (https://www.ncbi.nlm.nih.gov/Structure/bwrpsb/bwrpsb.cgi) (Yan et al., 2019) databases were used to manually confirm that the candidate sequences had complete TPS and TPP domains. The remaining genes were subsequently identified as members of the cucumber TPS family and were named according to their location on the cucumber's chromosome.

The length of open reading frames (ORFs) of cucumber TPS genes was predicted using the NCBI-ORFfinder (https://www.ncbi.nlm.nih.gov/orffinder/) website (Xie et al., 2015). The physicochemical properties and subcellular locations of the cucumber TPS proteins were forecast using Protparam (https://web.expasy.org/protparam/) (Zhang et al., 2019) and the Cell-PLoc2.0 (http://www.csbio.sjtu.edu.cn/bioinf/plant-multi/) website, respectively.

The cucumber TPS proteins' secondary structures were determined using the PRABI (https://npsa-prabi.ibcp.fr/cgi-bin/npsa_automat.pl?page=npsa_sopma.html) website (Xu et al., 2016).

\section{Phylogenetic analysis}

The phylogenetic tree, containing seven cucumber, eleven rice (Zang et al., 2011), eleven Arabidopsis (Yang et al., 2012), and twenty soybean (Xie et al., 2014) TPS protein sequences, was constructed based on multiple sequence alignments using Fasttree software and the maximum likelihood method. The bootstrap replication value was set as 1,000 and the other parameters remained constant. We used the evolview (https://evolgenius.info//evolviewv2/\#login) website to improve the appearance of the evolutionary tree. The TPS protein sequences of Arabidopsis, rice, and soybean were downloaded from TAIR (https://www.arabidopsis.org/) (Song et al., 2019), the Rice Genome Annotation Project 
133 (http://rice.plantbiology.msu.edu/) (Zang et al., 2011), and the Phytozome

134 (https://phytozome.jgi.doe.gov) (Yue et al. 2019) database, respectively (Supplementary file 1).

135

136

137

Gene structure, chromosomal location, and cis-acting element analysis

138

The GSDS2.0 (http://gsds.gao-lab.org/index.php) (Li et al., 2019) website was used to analyze

139 the gene structure of cucumber TPS genes and to plot the exon-intron diagram. We used the

140 TBtools software to combine the evolutionary tree with the gene structure diagram and Mapchart

141 software was applied to visualize the location of the genes on the chromosomes. The $2 \mathrm{~kb}$ sequences in the cucumber TPS genes' upstream region were screened as promoter sequences using Tbtools software. We used plantCARE (Song et al., 2019)

144 (http://bioinformatics.psb.ugent.be/webtools/plantcare/html/) to investigate the cis-acting elements in promoter regions to study the roles of genes in stress and hormone responses.

145

146

\section{Conserved motifs analysis}

147

We searched the MEME (http://meme-suite.org/) (Liu et al., 2019) website for conserved motifs of cucumber TPS proteins. The maximum retrieval value for the motif was set to 10 and the other parameters were set to default. InterProScan software was used to annotate the retrieved motifs.

151

152

153

154

155

156

157

158

159

160

161

162

163

164

165

166

167

168

169

170

171

\section{GO annotation}

We used the EggNOG mapper software (http://eggnog-mapper.embl.de/) to perform the gene ontology analysis. Cucumber TPS protein sequences were uploaded and Arabidopsis TPSs were used as the reference. GO analysis was categorized as: molecular function (MF), biological process (BP), and cellular component (CC).

\section{Plant materials, cultivation conditions, and treatments}

Cucumber seeds (C. sativus L. "Xin Chun 4") were germinated and grown in culture dishes with wet filter paper. Seedlings were transferred into hydroponic boxes once the cotyledon fully unfolded. The boxes contained Yamazaki cucumber nutrient solution and were placed in plant incubators at $25^{\circ} \mathrm{C}$, a light intensity of $200 \mu \mathrm{mol} \cdot \mathrm{m}^{-2} \mathrm{~s}^{-1}$, and a photoperiod of $14 \mathrm{~h} \mathrm{light} / 10 \mathrm{~h}$ dark (Niu et al., 2019). The nutrient solution was replaced every two days in order to maintain an adequate level of nutrients. Stress treatments were carried out when seedlings were at the twoleaf stage. Seedlings were grown in a $1 / 2$ nutrient solution containing $8 \%(\mathrm{w} / \mathrm{v}) \mathrm{PEG}, 50 \mathrm{mM}$ $\mathrm{NaCl}, 1 \mu \mathrm{M}$ IAA, $8 \%$ (w/v) $\mathrm{H}_{2} \mathrm{O}_{2}, 50 \mathrm{mM}$ sucrose, and $50 \mathrm{mM}$ mannitol, respectively, for drought, salt, IAA, $\mathrm{H}_{2} \mathrm{O}_{2}$, sucrose, and mannitol treatments. The concentrations of these reagents were determined by a preliminary experiment. Whole seedlings were frozen with liquid nitrogen after treatment at $0 \mathrm{~h}, 6 \mathrm{~h}, 12 \mathrm{~h}$ and $24 \mathrm{~h}$, and were stored at $-80^{\circ} \mathrm{C}$ (Zhu et al., 2019). The roots, stems, and leaves of untreated seedlings were collected at the two-leaf stage and were stored at $80^{\circ} \mathrm{C}$ to investigate the expression of cucumber TPS genes in the different tissues. Each 
172 treatment was performed with three biological replicates and each sample was collected from

173 five cucumber seedlings.

174

175

\section{RNA extraction, reverse transcription and quantitative real-time PCR}

176

The total RNA from different tissues and whole seedlings under different treatments were

177 extracted using the MiniBEST Plant RNA Extraction Kit (TaKaRa, Dalian, China). The RNA

178 concentration and purity were determined using the NaNo drop 1000 spectrophotometer and agarose gel electrophoresis (Xie et al., 2018). The FastQuant first strand cDNA synthesis kit (TIANGEN, Beijing, China) was used for the synthesis of cDNA following the manufacturer's protocol. The SuperReal PreMix Plus kit (TIANGEN, Beijing, China) and a Roche LightCycler instrument were used for qRT-PCR. The reaction system of qRT-PCR was as follows: $10 \mu \mathrm{L}$ $2 \times$ SuperReal PreMix Plus, $0.6 \mu \mathrm{L} 10 \mu \mathrm{M}$ forward primers, $0.6 \mu \mathrm{L} 10 \mu \mathrm{M}$ reverse primers, $2 \mu \mathrm{L}$ cDNA and $6.8 \mu \mathrm{L}$ RNase-free $\mathrm{ddH}_{2} \mathrm{O}$. The qRT-PCR procedure was as follow: $95^{\circ} \mathrm{C}$ for 15 minutes and 40 cycles of $95^{\circ} \mathrm{C}$ for 10 seconds and $60^{\circ} \mathrm{C}$ for 20 seconds. CsActin was used as an internal reference gene (Zhou et al., 2017). The primers of the cucumber TPS genes and CsActin for qRT-PCR were designed and synthesized using Sangon Biotech online software (Table 1). Three technical replicates were performed for each reaction.

189

\section{Statistical analysis}

191

The relative expression of the genes was calculated using the $2^{-\Delta \Delta C T}$ method (Han et al., 2020).

192 The expression of the CsTPS genes in the roots was used as a calibration sample to calculate their relative expressions in the stem and leaf (Zhang et al., 2014). The expression of CsTPS genes in untreated seedlings $(0 \mathrm{~h})$ was used as a calibration sample to calculate their relative expression at $6 \mathrm{~h}, 12 \mathrm{~h}$ and $24 \mathrm{~h}$. We adopted Duncan's ( $\mathrm{p}<0.05$ ) method for significance 196 analysis.

197

198

199

\section{Results}

200

\section{Genome-wide identification of TPS family in cucumber}

201

202

203

204

Seven TPS sequences were identified in the cucumber genome using bioinformatics methods.

The Pfam and NCBI-CDD databases were used for domain analysis to further prove the reliability of these candidate sequences. Our results indicated that all seven sequences had a typical TPS domain and belonged to the cucumber TPS family. The cucumber TPS genes

205 identified were named CSTPS1-CSTPS7 according to their location in the cucumber chromosome

206

207 (Table 2).

208

Domain analysis revealed that all cucumber TPS proteins contained a typical TPS

209

(Glyco_transf_20; Pfam: PF00982) domain in the N-terminal and a TPP (Trehalose_PPase; Pfam:

210 PF02358) domain in the C-terminal (Table 2). CsTPS3, CsTPS5, and CsTPS6 proteins all contained an additional Hydrolase-3 (Pfam: PF08282) domain (Supplementary file 2). 
212 Physical and chemical property analysis revealed that the length of the open reading frame of the 213 CsTPS genes was between 2,553 bp (CsTPS6) and 2,916 bp (CsTPS7); the length of cucumber 214 TPS proteins was between 850 (CsTPS6) and 971 (CsTPS7) amino acids; and their molecular 215 weight was between 96.31 KDa (CsTPS6) and 110.33 KDa (CsTPS7). The isoelectric point (pI) 216 ranged from 5.50 (CsTPS6) to 7.02 (CsTPS2). CsTPS2 was the only alkalescence (pI $>7$ ) in the 217 cucumber TPS proteins and the rest were acidic $(\mathrm{pI}<7)$ (Table 2).

218

219 The subcellular localization prediction indicated that cucumber TPS genes were mainly 220 distributed in the vacuole, chloroplast, and cytoplasm (Table 2).

221

222

The analysis of the secondary structure showed that cucumber TPS proteins were composed of

223 an $\alpha$-helix, random coil, extended strand, and a $\beta$-turn (Table 3 ).

224

225

226

\section{Phylogeny and gene structure analysis of CsTPSs}

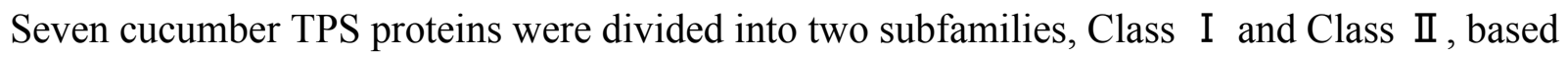

227 on the results of previous studies (Lunn, 2007; Mu et al., 2016). Among the seven CsTPS

228 proteins, CsTPS2 and CsTPS4 were classified as Class I and the remaining five proteins were classified as Class $I$ (Fig. 1A). In addition, the exon-intron diagram revealed that two members (CsTPS2 and CsTPS4) of Class I possessed 17 and 16 introns, respectively. In Class II, only CsTPS7 had six introns, while the other members all contained two introns (Fig. 1B). We inferred that two subfamilies went through functional differentiation in the course of evolution (Du et al., 2017).

234

We aligned the full-length protein sequences of seven cucumber TPS proteins, 11 Arabidopsis TPS proteins (Yang et al., 2012), 11 rice TPS proteins (Zang et al., 2011), and 20 soybean TPS proteins (Xie et al., 2014) to establish a maximum likelihood phylogenetic tree (Fig. 2) to further investigate the evolutionary relationships of TPS family in various species. As previously described, the TPSs in cucumber, Arabidopsis, rice, and soybean may be differentiated into two subfamilies: Class I and Class $\mathbb{I}$. The two subfamilies were further divided into seven subgroups: I -1, I -2, II-1, II-2, II-3, II-4 and II-5, based on the phylogenetic relationship with high bootstrap support (Xie et al., 2015). Subgroup I -1 contained nine members originating from Arabidopsis (1), rice (1), cucumber (2), and soybean (5), respectively. I -2 contained three members which all originated from Arabidopsis. $\mathbb{I}$-1 contained nine members which were derived from Arabidopsis (2), rice (2), cucumber (1), and soybean (4), respectively. II -2 contained four members which derived from cucumber (1) and soybean (3), respectively. II -3 contained ten members which originated from Arabidopsis (3), rice (2), cucumber (1), and soybean (4), respectively. II -4 contained six members which originated from Arabidopsis (1), 
249

250

251

252

253

254

255

256

257

258

259

260

261

262

263

264

265

266

267

268

269

270

271

272

273

274

275

276

277

278

279

280

281

282

283

284

285

286

287

288

rice (3), and soybean (2), respectively. II -5 contained eight members which derived from Arabidopsis (1), rice (3), cucumber (2), and soybean (2), respectively. With the exception of I 2 , $\amalg-2$, and $\amalg-4$, the other subgroups contained at least one member of four species. Our results showed that some of the TPS proteins of cucumber and soybean were divided into the same subgroup, indicating that they were closely related. In addition, previous studies have shown that most of TPS genes in the Class I had 16 introns, while TPS genes in the Class II possessed 2 introns (Yang et al. 2012). When combined with the analysis of the cucumber TPS gene structure, we can speculate that the genes of the same group were close to each other during the evolution process, while the genes of different groups were far away from each other during the evolution process (Xie et al., 2015).

\section{Conserved motifs of cucumber TPS proteins}

We used the MEME online website to study the characteristic regions of cucumber TPS proteins. We searched 10 conserved motifs in cucumber TPS proteins (Fig. 3). The lengths of these conserved motifs were between 29 and 50 amino acids and the sequence information of these 10 conserved motifs is listed in Table 4. Cucumber TPS proteins classified into the same subfamily in the evolutionary tree possessed a similar or identical motif composition (Ou et al., 2018). For instance, members of Class $\mathbb{I}$ possessed all 10 conserved motifs, while members (CsTPS2 and CsTPS4) of Class I lacked motifs 8 and 9. Motifs 4, 5, 1, 9, 6, 7, and 3 together constituted the TPS domain and motifs 10, 2, and 8 constituted the TPP domain, according to annotation. These results further supported the reliability of the phylogenetic classification of cucumber TPS proteins and indicated that the two subfamilies may have produced a functional difference during evolution (Xie et al., 2018).

\section{Chromosomal location of cucumber TPS genes}

We used Mapchart software to analyze the location of CsTPS genes on cucumber chromosomes. The results showed that seven CSTPS genes were randomly located on six cucumber chromosomes (Fig. 4). Two genes (CsTPS1 and CsTPS2) were located on chromosome 1, while only single genes existed on the other chromosomes. The majority of the CsTPS genes were located on the proximate or distal ends of the cucumber chromosomes (Zhao et al., 2018).

\section{Cis-acting element analysis of cucumber TPS genes}

A total of 72 types of elements were found in the promoter regions of the CsTPS genes. All CsTPS genes contained CAAT and TATA boxes in the promoter regions, which were core and common promoter elements (Zhou et al., 2017) (Supplementary file 3). The cis-acting elements were identified and categorized as stress-related elements and plant hormone-responsive elements (Zhu et al., 2019). Stress-related elements, including MYB (stress response element), MYC (the recognition site of cold-resistant element), and ARE (anaerobic induction element) were mainly found in promoter regions of most CsTPS genes (Zhou et al., 2017). Some plant hormone-responsive elements, including ERE (ethylene-responsive element), ABRE (abscisic

PeerJ reviewing PDF | (2020:12:56113:2:0:NEW 10 Apr 2021) 
289

290

291

292

293

294

295

296

297

298

299

300

301

302

303

304

305

306

307

308

309

310

311

312

313

314

315

316

317

318

319

320

321

322

323

324

325

326

327

328

acid responsive element), TCA-element (salicylic acid-responsive element), and TGA-element (auxin-responsive element) (Han et al., 2020) were also found in most CsTPS genes (Fig. 5 and Fig. 6). Light responsive elements including Box4, AE-box, G-Box, and GATA-motif were widely found in promoter regions of cucumber TPS genes (Fig. 5 and Supplementary file 3). Our results indicated that TPS genes played a significant role in stress, hormone, and light response (Zhao et al., 2018).

\section{GO annotation of cucumber TPS proteins}

We found that a majority of cucumber TPS proteins participated in UDP-glycosyltransferase activity, hydrolase activity, phosphatase activity, catalytic activity, and alpha-trehalosephosphate synthase activity (Supplementary file 4). CsTPS2 and CsTPS4 were involved in transferase activity and phosphoric ester hydrolase activity. Cellular component analysis showed that most CsTPS proteins were located on the cytoplasm and cytosol. However, CsTPS2 and CsTPS4 were also located intracellularly. Biological process analysis indicated that most CsTPS proteins were involved in various biological processes, including metabolism, biosynthesis, cellular processes, and development. Some CsTPS proteins also participated in the stress response, signal transduction, post-embryonic development, and seed, fruit, and reproductive system development.

\section{Expression analysis of cucumber TPS genes in different tissues}

We measured the expression of CsTPS genes in root, stem, and leaf using qRT-PCR to determine the expression specificity of cucumber TPS genes in different tissues. The expression of CsTPS genes was detected in the root, stem, and leaf (Fig 7) and their expressions were high, moderate, and low in the root, leaf, and stem, respectively. Our results indicated that CSTPS genes may play a specific role in the growth of cucumber seedlings.

\section{Expression analysis of cucumber TPS genes under different treatments}

We conducted qRT-PCR experiments and drew a cluster heatmap to determine the expression patterns of CsTPS genes under various treatments (Fig. 8). Our results indicated different patterns for the expression of CSTPS genes with various treatments. Under PEG, the expression of CSTPS3 was up-regulated significantly and peaked at $24 \mathrm{~h}$ and CSTPS2 and CSTPS5 were upregulated slightly. However, CsTPS1, CsTPS4, CsTPS6 and CsTPS7 were down-regulated and reached their lowest expression levels at $24 \mathrm{~h}$. The expression of CSTPS3 and CsTPS4 was upregulated significantly with $\mathrm{NaCl}$ treatment and reached the highest expression level at $24 \mathrm{~h}$; the expression of CsTPS2 and CSTPS7 also increased. However, CsTPS1 and CsTPS6 were downregulated with the $\mathrm{NaCl}$ treatment and reached the lowest expression level at $24 \mathrm{~h}$. Under $\mathrm{H}_{2} \mathrm{O}_{2}$ treatment, CsTPS1 and CsTPS5 were activated while others genes were inhibited (CsTPS2, CsTPS3, CsTPS4 and CsTPS6) or did not show obvious trends (CsTPS7). Under mannitol treatment, CSTPS3 and CSTPS7 were up-regulated with the highest expression level at $24 \mathrm{~h}$ and CsTPS1 and CsTPS4 were slightly induced. Conversely, the mannitol treatment caused a large 
329

330

331

332

333

334

335

336

337

338

339

340

341

342

343

344

345

346

347

348

349

350

351

352

353

354

355

356

357

358

359

360

361

362

363

364

365

366

decline in the expression of CsTPS2, CsTPS5 and CsTPS6. Under IAA treatment, CsTPS3 and CsTPS4 were up-regulated significantly, whereas others genes showed no clear trends. CsTPS3 and CsTPS4 showed a strong expression under sucrose treatment, whereas CsTPS1 and CsTPS7 were induced slightly by sucrose. CsTPS2, CsTPS5 and CsTPS6 were inhibited by sucrose. These results showed that CSTPS genes may be involved in stress, hormone, and sugar responses.

\section{Discussion}

Trehalose-6-phosphate synthase (TPS) is vital for the stress response and trehalose metabolism. Genes encoding TPS have been identified in many plants in the form of a gene family (Avonce et al., 2014). Previous studies have found 11, 11, 11, 8, 20, 13, 12, 10, 9, and 53 TPS genes in Arabidopsis (Yang et al., 2012), rice (Zang et al., 2011), pepper (Wei et al., 2016), potato (Xu et al., 2017), soybean (Xie et al., 2014), apple (Du et al., 2017), winter wheat (Xie et al., 2015), tomato (Chen et al., 2017), sugarcane (Hu et al., 2020), and cotton (Mu et al., 2016), respectively. However, the TPS gene family in cucumber has not been well-studied. We identified seven TPS genes in the cucumber genome, which were randomly located in six chromosomes of cucumber. Differences in the genome size of different species may cause variations in the number of members of the TPS family (Xie et al., 2018).

Cucumber TPS genes were divided into two subfamilies: Class I (CsTPS2 and CsTPS4) and Class II, which was consistent with the classification in Arabidopsis (Yang et al., 2012), rice (Zang et al., 2011) and pepper (Wei et al., 2016). We found that four (AtTPS1-AtTPS4), one (OsTPS1), and three (GaTPS1-GaTPS3) TPS genes belonged to Class I in Arabidopsis, rice, and pepper, respectively. Class I genes in our study had 16-17 introns, while Class II genes had 2 introns, with the exception of CsTPS7. Motif analysis showed that Class II genes possessed all 10 conserved motifs, whereas Class I genes lacked motif 8 and 9 in cucumber. These results were supported by results from earlier studies in Arabidopsis (Yang et al., 2012), rice (Zang et al., 2011), and cotton (Mu et al., 2016). Class I genes possessed 16 introns in Arabidopsis and rice, whereas Class II genes had 2 introns. Class I genes in cotton had more introns than Class II genes and lacked motif 8 (Mu et al., 2016). According to a previous study, three mechanisms (exon/intron gain/loss, exonization/pseudoexonization, and insertion/deletion) may cause the difference in gene structure (Xu et al., 2012). These studies illustrated that the two subfamilies may have experienced functional differentiation during evolution (Xie et al., 2018). Domain analysis indicated that all cucumber TPS genes possessed a TPS domain at the Nterminal and a TPP domain at the C-terminal, which was consistent with results from earlier studies in Arabidopsis (Yang et al., 2012), pepper (Wei et al., 2016), and apple (Du et al., 2017). CsTPS3, CsTPS5, and CsTPS6 proteins all contained a Hydrolase-3 (Pfam: PF08282) domain. However, some TPS genes lacked either a TPS domain or a TPP domain; GrTPS6, GhTPS4, and 
367

368

369

370

371

372

373

374

375

376

377

378

379

380

381

382

383

384

385

386

387

388

389

390

391

392

393

394

395

396

397

398

399

400

401

402

403

404

405

406

GhTPS9 genes in cotton lacked a TPP domain (Mu et al., 2016) and the loss of the domain may be the result of evolution. Evolutionary analysis showed that 49 TPS proteins from Arabidopsis, rice, cucumber, and soybean were divided into two subfamilies and they were further classified into seven subgroups, similar to the classifications from a previous study (Xie et al., 2015). The CsTPS proteins were grouped together with at least one TPS protein from other species in the evolutionary tree, indicating that TPS proteins from different species had similar functions (Zhou et al., 2017).

Cis-acting elements were involved in the regulation of gene expression. Certain transcription factors have been shown to be activated and combined with cis-acting elements to activate the expression of stress-related genes when plants were exposed to adverse conditions (Hadiarto et al., 2011). We found that some elements related to stress (MBS, LTR, ARE), hormones (ABRE, ERE, TCA-element), and light response (AE-box, Box 4, TCT-motif) existed widely in promoter regions of most cucumber TPS genes. These elements consistently appeared in potato TPS genes (Xu et al., 2017), indicating that TPS genes may be involved in the stress, hormone, and light responses. The overexpression of TPS genes in Arabidopsis, rice, and potato improved their stress tolerance (Avonce et al., 2004, Li et al., 2011, Kondrák et al., 2011). Our study showed that CsTPS3 was significantly induced by drought stress, which was consistent with a study in Arabidopsis (Avonce et al., 2004). CSTPS3 and CSTPS4 showed strong expression under salt stress, which was in agreement with the results of OSTPS1 (Li et al., 2011). CsTPS1 and CsTPS5 showed strong expression under oxidative stress. CSTPS3 and CSTPS7 were clearly induced by osmotic stress by mannitol, which coincides with the results from cotton and watermelon (Mu et al., 2016). CsTPS3 was induced significantly by drought, salt and osmotic stresses, indicating that CsTPS3 may be more sensitive to various abiotic stress than other CsTPS genes. Plant hormones play an important role in signal transduction, plant growth, and development. We found that CsTPS3 and CsTPS4 were up-regulated by IAA, while other CsTPS genes were down-regulated by IAA, which were similar to the results for potato (Xu et al., 2017). GO analysis indicated that CsTPS proteins may participate in the response to stress, which further supported our results.

Studies have shown that AtTPS1 participated in embryonic development and vegetative growth through the ABA mechanism and sugar metabolism (Gómez et al., 2010). AtTPS1 played an essential role in regulating sugar signaling (Avonce et al., 2004) and Arabidopsis TPSs could be repressed or induced by sugar. We found that CSTPS3 and CSTPS4 were significantly induced by sucrose, while CsTPS2, CsTPS5, and CsTPS6 were repressed by sucrose. CsTPS3 was highly sensitive to abiotic stress (except oxidative stress), hormone, and sucrose treatments. In contrast, abiotic stress, hormone, and sucrose treatments caused a large decline in the expression of CsTPS6. The expression analysis of CsTPS genes in various tissues showed that CsTPS genes were expressed in the root, stem, and leaf and had the highest expression levels in the root. The expression of most TPS genes in cotton was induced by low temperature, salt, and drought, while

Peer) reviewing PDF | (2020:12:56113:2:0:NEW 10 Apr 2021) 
407 their expression patterns were different (Mu et al., 2016). In winter wheat, TaTPS1 and TaTPS3 408 expression was up-regulated under a freeze treatment $\left(-20^{\circ} \mathrm{C}\right)$ (Xie et al., 2015). In potato, the 409 expression and patterns of StTPS genes were regulated by different stresses (salt, heat and 410 osmotic) and hormones (IAA, ABA and $\mathrm{GA}_{3}$ ) (Xu et al., 2017). In maize, the expression of $411 Z m T P S$ genes was induced under salt and low temperature stresses (Jiang et al., 2010). In tomato, 412 the expression of SITPS1 was inhibited by red and blue light (Chen et al., 2017). These results 413 indicate that TPS genes may play critical roles in responding to stress, hormones, and light.

414

415

416

417

418

419

420

421

422

423

424

425

426

427

428

429

430

431

432

433

434

435

436

437

438

439

440

441

442

443

Trehalose may be involved in plant stress resistance including salt, cold, drought, and heavy metal. TPS is a key enzyme of trehalose metabolism with an essential role in plant stress resistance. Related studies have shown that the overexpression of TPS genes improved the tolerance of plants under unfavorable conditions (Li et al., 2011, Kondrák et al., 2011, Liu et al., 2019). Only one or two genes classified as Class I encoded active trehalose-6-phosphate synthase (TPS) in most species. In rice, only proteins encoded by OsTPS1 had TPS activity and all TPS proteins had no TPP activity (Zang et al., 2011). In Arabidopsis, only AtTPS1 (encoded by Class I genes) had TPS activity but no TPP activity, while TPS proteins encoded by Class II genes and remaining Class I genes had neither TPS nor TPP activity (Yang et al., 2012). In maize, ZmTPS1 possessed TPS activity (Jiang et al., 2010). We believe that CsTPS2 and CsTPS4 have TPS activity, but this requires further experimental support. Related studies have indicated that the accumulation of harmful mutations and changes in protein conformation may lead to the loss of TPS activity (Yang et al., 2012, Vandesteene et al., 2010). Previous studies have shown that the trehalose pathway was essential in regulating the use and distribution of sucrose, coordinating source-sink relation, the effective utilization of carbohydrates (Schluepmann et al., 2004), and improving crop yield (Paul et al., 2020). Studies in cucumber fruit showed that there was a strong correlation between T6P and sucrose (Zhang et al., 2015). The role of the trehalose pathway in reproductive growth and sugar metabolism should be the focus of future research.

\section{Conclusion}

We identified seven TPS genes in the cucumber genome and analyzed their physicochemical properties, gene structures, domains, conserved motifs, evolutionary relationships, gene locations, cis-elements, GO analysis, and expression patterns. Our results demonstrated that cucumber TPS genes play an important role in the response to stresses, sucrose, and phytohormones. Our study provides reference information for future studies of the mechanisms of TPS proteins on the growth, development, stress-resistance, and trehalose pathway in cucumber. 
444

445

446

447

448

449

450

451

452

453

454

455

456

457

458

459

460

461

462

463

464

465

466

467

468

469

470

471

472

473

474

475

476

477

478

479

480

481

482

483

\section{References}

Abdallah MM, Abdelgawad ZA, El-bassiouny HMS (2016) Alleviation of the adverse effects of salinity stress using trehalose in two rice varieties. S. Afr. J. Bot. 103, 275-282. doi: 10.1016/j.sajb.2015.09.019

Abdallah MM, El Sebai TN, Ramadan AAE, El-Bassiouny HMS (2020) Physiological and biochemical role of proline, trehalose, and compost on enhancing salinity tolerance of quinoa plant. Bulletin of the National Research Centre. 44, 96. doi: 10.1186/s42269-02000354-4

Ashraf M, Foolad MR (2007) Roles of glycine betaine and proline in improving plant abiotic stress resistance. Environ. Exp. Bot. 59 (2), 206-216. doi: 10.1016/j.envexpbot.2005.12.006

Avonce N, Leyman B, Mascorro-Gallardo JO, Van Dijck P, Thevelein JM, Iturriaga G (2004) The Arabidopsis trehalose-6-P synthase AtTPS1 gene is a regulator of glucose, abscisic acid, and stress signaling. Plant Physiol. 136 (3), 3649-3659. doi: 10.1104/pp.104.052084

Avonce N, Leyman B, Mascorro-Gallardo JO, Van Dijck P, Thevelein JM, Iturriaga G (2004) The Arabidopsis trehalose-6-P synthase AtTPS1 gene is a regulator of glucose, abscisic acid, and stress signaling. Plant Physiol. 136(3), 3649-59. doi: 10.1104/pp.104.052084

Baninasab B (2010) Induction of drought tolerance by salicylic acid in seedlings of cucumber (Cucumis sativus L.). J. Hortic. Sci. Biotech. 85 (3), 191-196. doi: 10.1080/14620316.2010.11512653

Chen Q, Chen QJ, Sun GQ, Zheng K, Yao ZP, Han YH, Wang LP, Duan YJ, Yu DQ, Qu YY (2019) Genome-Wide Identification of Cyclophilin Gene Family in Cotton and Expression Analysis of the Fibre Development in Gossypium barbadense. Int J Mol Sci. 20 (2), 349. doi: 10.3390/ijms20020349

Chen ZX, Lou J (2017) Identification and expression of the trehalose-6-phosphate synthase gene family members in tomato exposed to different light spectra. Arch Biol Sci. 69 (1), 93-101.

Cheng C, Wang Y, Chai FM, Li SH, Xin HP (2018) Genome-wide identification and characterization of the 14-3-3 family in Vitis vinifera L. during berry development and coldand heat-stress response. BMC Genom. 19, 579. doi: 10.1186/s12864-018-4955-8

Deinlein U, Stephan AB, Horie T, Luo W, Xu G, Schroeder JI (2014) Plant salt-tolerance mechanisms. Trends Plant Sci. 19 (6), 371-379. doi: 10.1016/j.tplants.2014.02.001 doi: 10.2298/ABS160325082C

Du LS, Qi SY, Ma JJ, Xing LB, Fan S, Zhang SW, Li YM, Zhang D, Han MY (2017) Identification of TPS family members in apple (Malus $x$ domestica Borkh.) and the effect of sucrose sprays on TPS expression and floral induction. Plant Physiol. Biochem. 120, 10-23. doi: 10.1016/j.plaphy.2017.09.015

Elbashiti T, Hamamci H, Oktem HA, Yucel M (2005) Biochemical analysis of trehalose and its metabolizing enzymes in wheat under abiotic stress conditions. Plant Sci. 169 (1), 47-54. doi: 10.1016/j.plantsci.2005.02.024

Elbein AD, Pan YT, Pastuszak I, Carroll D (2003) New insights on trehalose: a multifunctional 
484

485

486

487

488

489

490

491

492

493

494

495

496

497

498

499

500

501

502

503

504

505

506

507

508

509

510

511

512

513

514

515

516

517

518

519

520

521

522

523

molecule. Glycobiology. 13 (4), 17-27. doi: 10.1093/glycob/cwg047

Fichtner F, Olas JJ, Feil R, Watanabe M, Krause U, Hoefgen R, Stitt M, Lunn JE (2020) Functional Features of TREHALOSE-6-PHOSPHATE SYNTHASE1, an Essential Enzyme in Arabidopsis. The Plant Cell. 32 (6), 1949-1972. doi: 10.1105/tpc.19.00837

Geonhee H, Sara K, Jae-Yong C, Inyup P, Jeong K, Eunkyoo O (2019) Trehalose-6-phosphate signaling regulates thermoresponsive hypocotyl growth in Arabidopsis thaliana. EMBO reports. 20 (10), e47828. doi: 10.15252/embr.201947828

Goddijn OJ, van Dun K (1999) Trehalose metabolism in plants. Trends Plant Sci. 4 (8), 315319. doi: 10.1016/S1360-1385(99)01446-6

Gómez LD, Gilday A, Feil R, Lunn JE, Graham IA (2010) AtTPS1-mediated trehalose 6-phosphate synthesis is essential for embryogenic and vegetative growth and responsiveness to ABA in germinating seeds and stomatal guard cells. The Plant Journal. 64, 1-13. doi:10.1111/j.1365-313X.2010.04312.x

Griffiths CA, Sagar R, Geng YQ, Primavesi LF, Patel MK, Passarelli MK, Gilmore IS, Steven RT, Bunch J, Paul MJ, Davis BG (2016) Chemical intervention in plant sugar signalling increases yield and resilience. Nature. 540(7634), 574-578. doi: 10.1038/nature20591

Hadiarto T, Tran LS (2011) Progress studies of drought-responsive genes in rice. Plant Cell Rep. 30 (3), 297-310. doi: 10.1007/s00299-010-0956-Z

Han LM, Hua WP, Cao XY, Yan JA, Chen C, Wang ZZ (2020) Genome-wide identification and expression analysis of the superoxide dismutase (SOD) gene family in Salvia miltiorrhiza. Gene. 144603. doi: 10.1016/j.gene.2020.144603

Hightower LE (1991) Heat shock, stress proteins, chaperones, and proteotoxicity. Cell. 66 (2), 191-197. doi: 10.1016/0092-8674(91)90611-2

$\mathrm{Hu}$ X, Wu ZD, Luo ZY, Burner DM, Pan YB, Wu CW (2020) Genome-Wide Analysis of the Trehalose-6-Phosphate Synthase (TPS) Gene Family and Expression Profiling of ScTPS Genes in Sugarcane. Agronomy. 10 (7), 969. doi: 10.3390/agronomy10070969

Jiang W, Fu FL, Zhang SZ, Wu L, Li WC (2010) Cloning and Characterization of Functional Trehalose-6-Phosphate Synthase Gene in Maize. J Plant Biol. 53, 134-141. doi: 0.1007/s12374-010-9098-7

Kim TH 2012 Plant stress surveillance monitored by ABA and disease signaling interactions. Mol Cells. 33 (1), 1-7. doi: 10.1007/s10059-012-2299-9

Kondrák M, Marincs F, Kalapos B, Juhász Z, Bánfalvi Z (2011) Transcriptome analysis of potato leaves expressing the trehalose-6-phosphate synthase 1 gene of yeast. PLoS One. 6 (8), e23466. doi: 10.1371/journal.pone.0023466

Li HW, Zang BS, Deng XW, Wang XP (2011) Overexpression of the trehalose-6-phosphate synthase gene OsTPS1 enhances abiotic stress tolerance in rice. Planta. 234, 1007-1018. doi: 10.1007/s00425-011-1458-0

Li HY, Xu GZ, Yang C, Yang L, Liang ZH (2019) Genome-wide identification and expression analysis of HKT transcription factor under salt stress in nine plant species. Ecotoxicol. Environ. Saf. 171, 435-442. doi: 10.1016/j.ecoenv.2019.01.008 
524

525

526

527

528

529

530

531

532

533

534

535

536

537

538

539

540

541

542

543

544

545

546

547

548

549

550

551

552

553

554

555

556

557

558

559

560

561

562

563

Li JM, Zhang MH, Sun J, Mao XR, Wang J, Wang JG, Liu HL, Zheng HL, Zhen Z, Zhao HW, Zou DT (2019) Genome-Wide Characterization and Identification of Trihelix Transcription Factor and Expression Profiling in Response to Abiotic Stresses in Rice (Oryza sativa L.). Int J Mol Sci. 20 (2), 251. doi: 10.3390/ijms20020251

Liu MY, Fu QK, Ma ZT, Sun WJ, Huang L, Wu Q, Tang ZZ, Bu TL (2019) Genome-wide investigation of the MADS gene family and dehulling genes in tartary buckwheat (Fagopyrum tataricum). Planta. 249, 1301-1318. doi: 10.1007/s00425-019-03089-3

Liu X, Fu LS, Qin P, Sun YL, Liu J, Wang X (2019) Overexpression of the wheat trehalose 6phosphate synthase 11 gene enhances cold tolerance in Arabidopsis thaliana. Gene. 710, 210-217. doi: 10.1016/j.gene.2019.06.006

Liu Y, Wen HS, Qi X, Zhang XY, Zhang KQ, Fan HY, Tian Y, Hu YB, Li Y (2019) Genomewide identification of the $\mathrm{Na}+/ \mathrm{H}+$ exchanger gene family in Lateolabrax maculatus and its involvement in salinity regulation. Comp Biochem Phys D. 29, 286-298. doi: 10.1016/j.cbd.2019.01.001

Lunn JE (2007) Gene families and evolution of trehalose metabolism in plants. Functional Plant Biology. 34 (6), 550-563. doi: 10.1071/FP06315

Lunn JE, Delorge I, Figueroa CM, Dijck PV, Stitt M (2015) Trehalose metabolism in plants. Plant J. 79 (4), 544-567. doi: 10.1111/tpj.12509

Miao YX, Luo XY, Gao XX, Wang WJ, Bin L, Hou LP (2020) Exogenous salicylic acid alleviates salt stress by improving leaf photosynthesis and root system architecture in cucumber seedlings. Sci. Hortic. 109577. doi: 10.1016/j.scienta.2020.109577

Mu M, Lu XK, Wang JJ, Wang DL, Yin ZJ, Wang S, Fan WL, Ye WW (2016) Genome-wide Identification and analysis of the stress-resistance function of the TPS (Trehalose-6Phosphate Synthase) gene family in cotton. BMC Genet. 17 (1), 54. doi: 10.1186/s12863016-0360-y

Niu LJ, Yu JH, Liao WB, Xie JM, Yu J, Xiao XM, Hu LL, Wu Y (2019) Proteomic Investigation of S-Nitrosylated Proteins During NO-Induced Adventitious Rooting of Cucumber. Int J Mol Sci. 20 (21), 5363. doi: 10.3390/ijms20215363

Nuccio ML, Wu J, Mowers R, Zhou HP, Meghji M, Primavesi LF, Paul MJ, Chen X, Gao Y, Haque E, Basu SS, Lagrimini LM (2015) Expression of trehalose-6-phosphate phosphatase in maize ears improves yield in well-watered and drought conditions. Nat Biotechnol. 33, 862-869. doi: 10.1038/nbt.3277

Ou WJ, Mao X, Huang C, Tie WW, Yan Y, Ding ZH, Wu CL, Xia ZQ, Wang WQ, Zhou SY, Li KM, Hu W (2018) Genome-Wide Identification and Expression Analysis of the KUP Family under Abiotic Stress in Cassava (Manihot esculenta Crantz). Front Physiol. 9, 17. doi: 10.3389/fphys.2018.00017

Paul MJ, Watson A, Griffiths CA (2020) Trehalose 6-phosphate signalling and impact on crop yield. Biochem Soc Trans. 48 (5), 2127-2137. doi: 10.1042/BST20200286

Schluepmann H, Pellny T, van Dijken A, Smeekens S, Paul M (2003) Trehalose 6-phosphate is indispensable for carbohydrate utilization and growth in Arabidopsis thaliana. PNAS. 100 
564

565

566

567

568

569

570

571

572

573

574

575

576

577

578

579

580

581

582

583

584

585

586

587

588

589

590

591

592

593

594

595

596

597

598

599

600

601

602

603

\section{(11), 6849-6854. doi: 10.1073/pnas.1132018100}

Smeekens S (2017) Drought resistance: Spraying for yield. Nat. Plants. 3, 17-23. doi: 10.1038/nplants.2017.23

Song M, Peng XY (2019) Genome-Wide Identification and Characterization of DIR Genes in Medicago truncatula. Biochem. Genet. 57 (4), 487-506. doi: 10.1007/s10528-019-09903-7

Song ZP, Pan FL, Lou XP, Wang DB, Yang C, Zhang BQ, Zhang HY (2019) Genome-wide identification and characterization of Hsp70 gene family in Nicotiana tabacum. Mol. Biol. Rep. 46 (2), 1941-1954. doi: 10.1007/s11033-019-04644-7

Vandesteene L, Ramon M, Le Roy K, Van Dijck P, Rolland F (2010) A single active trehalose6-P synthase (TPS) and a family of putative regulatory TPS-like proteins in Arabidopsis. Mol. Plant. 3 (2), 406-419. doi: 10.1093/mp/ssp114

Wahl V, Ponnu J, Schlereth A, Arrivault S, Langenecker T, Franke A, Feil R, Lunn JE, Stitt M, Schmidet M (2013) Regulation of Flowering by Trehalose-6-Phosphate Signaling in Arabidopsis thaliana. Science. 339(6120), 704-707. doi: 10.1126/science.1230406

Wang K, Li FJ, Gao ML, Huang YC, Song ZG (2020) Mechanisms of trehalose-mediated mitigation of $\mathrm{Cd}$ toxicity in rice seedlings. J. Clean. Prod. 121982. doi: 10.1016/j.jclepro.2020.121982

Wang RQ, Zhao P, Kong NN, Lu RZ, Pei Y, Huang M, Chen Q (2018) Genome-Wide Identification and Characterization of the Potato bHLH Transcription Factor Family. Genes. 9 (1), 54. doi: 10.3390/genes9010054

Wei BQ, Wang LL, Zhang R, Chen LZ, Zhang SL, Zhang JN (2016) Identification of CaTPS gene family and expression analysis of CaTPS1 in hot pepper. Acta Horticulturae Sinica. 43 (8), 1504-1512. doi: 10.16420/j.issn.0513-353x.2016-0207

Xie DW, Wang XN, Fu LS, Sun J, Zheng W, Li ZF (2015) Identification of the trehalose-6phosphate synthase gene family in winter wheat and expression analysis under conditions of freezing stress. J. Genet. 94 (1), 55-65. doi: 10.1007/s12041-015-0495-z

Xie L, Wang ZX, Huang B (2014) Genome-wide identification classification and expression of TPS family genes in soybean. Chinese Journal of Oil Crop Sciences. 36 (2) 160-167. doi: 10.7505/j.issn.1007-9084.2014.02.004

Xie T, Chen CJ, Li CH, Liu JR, Liu CY, He YH (2018) Genome-wide investigation of WRKY gene family in pineapple: evolution and expression profiles during development and stress. BMC Genom. 19 (1), 490. doi: 10.1186/s12864-018-4880-x

Xu GX, Guo C, Shan HY, Kong HZ (2012) Divergence of duplicate genes in exon-intron structure. Proc Natl Acad Sci USA. 109 (4), 1187-1192. doi: 10.1073/pnas.1109047109

$\mathrm{Xu}$ JN, Xing SS, Cui HR, Chen XS, Wang XY (2016) Genome-wide identification and characterization of the apple (Malus domestica) HECT ubiquitin-protein ligase family and expression analysis of their responsiveness to abiotic stresses. Mol Genet Genomics. 291 (2), 635-646. doi: 10.1007/s00438-015-1129-0

Xu YC, Wang YJ, Mattson N, Yang L, Jin QJ (2017) Genome-wide analysis of the Solanum tuberosum (potato) trehalose-6-phosphate synthase (TPS) gene family: evolution and 
604

605

606

607

608

609

610

611

612

613

614

615

616

617

618

619

620

621

622

623

624

625

626

627

628

629

630

631

632

633

634

635

636

637

638

639

640

641

642

643

differential expression during development and stress. BMC Genom. 18 (1), 926. doi: 10.1186/s12864-017-4298-x

Yadav UP, Ivakov A, Feil R, Duan GY, Walther D, Giavalisco P, Piques M, Carillo P, Hubberten HM, Stitt M, Lunn JE (2014) The sucrose-trehalose 6-phosphate (Tre6P) nexus: specificity and mechanisms of sucrose signalling by Tre6P. J EXP BOT. 65(4), 1051-1068, doi: https: 10.1093/jxb/ert457

Yan F, Li HZ, Zhao P (2019) Genome-Wide Identification and Transcriptional Expression of the PAL Gene Family in Common Walnut (Juglans Regia L.). Genes. 10 (1), 46. doi: 10.3390/genes 10010046

Yang HL, Liu YJ, Wang CL, Zeng QY (2012) Molecular Evolution of Trehalose-6-Phosphate Synthase (TPS) Gene Family in Populus, Arabidopsis and Rice. PLOS ONE. 7 (8), e42438. doi: 10.1371/journal.pone.0042438

Yue H, Chang X, Zhi YQ, Wang L, Xing GW, Song WN, Nie XJ (2019) Evolution and Identification of the WRKY Gene Family in Quinoa (Chenopodium quinoa). Genes. 10 (2), 131. doi: 10.3390/genes10020131

Zang BS, Li HW, Li WJ, Deng XW, Wang XP (2011) Analysis of trehalose-6-phosphate synthase (TPS) gene family suggests the formation of TPS complexes in rice. Plant Mol. Biol. 76 (6), 507-522. doi: 10.1007/s11103-011-9781-1

Zhang LS, Wu B, Zhao DG, Li CL, Shao FJ, Lu SF (2014) Genome-wide analysis and molecular dissection of the SPL gene family in Salvia miltiorrhiza [J]. J Integr Plant Biol. 56(01), 3850. doi: 10.1111/jipb.12111

Zhang W, Wang SY, Yu FW, Tang J, Yu L, Wang H, Li JB (2019) Genome-Wide Identification and Expression Profiling of Sugar Transporter Protein (STP) Family Genes in Cabbage (Brassica oleracea var. capitata L.) Reveals their Involvement in Clubroot Disease Responses. Genes. 10 (1), 71. doi: 10.3390/genes10010071

Zhang ZP, Deng Y, Song X, Miao M (2015) Trehalose-6-phosphate and SNF1-related Protein Kinase 1 are Involved in the First-fruit Inhibition of Cucumber. J PLANT PHYSIOL. 177, 110-120. doi: 10.1016/j.jplph.2014.09.009

Zhao P, Wang DD, Wang RQ, Kong NN, Zhang C, Yang CH, Wu WT, Ma HL, Chen Q (2018) Genome-wide analysis of the potato Hsp20 gene family: identification, genomic organization and expression profiles in response to heat stress. BMC Genom. 19 (1), 61. doi: 10.1186/s12864-018-4443-1

Zhou Y, Hu LF, Wu H, Jiang LW, Liu SQ, Pesole G (2017) Genome-Wide Identification and Transcriptional Expression Analysis of Cucumber Superoxide Dismutase (SOD) Family in Response to Various Abiotic Stresses. Int J Genomics. 1-14. doi: 10.1155/2017/7243973

Zhu JK (2016) Abiotic Stress Signaling and Responses in Plants. Cell. 167 (2), 313-324. doi: 10.1016/j.cell.2016.08.029

Zhu YX, Yang L, Liu N, Yang J, Zhou XK, Xia YC, He Y, He YQ, Gong HJ, Ma DF, Yin JL (2019) Genome-wide identification, structure characterization, and expression pattern profiling of aquaporin gene family in cucumber. BMC Plant Biol. 19 (1), 345. doi: 
$644 \quad 10.1186 / \mathrm{s} 12870-019-1953-1$ 


\section{Figure 1}

Phylogenetic relationships and gene structures of CSTPSs.

(A) The evolutionary tree was built based on the full-length cucumber TPS protein sequences using Fasttree software. (B) The exon-intron diagram of cucumber TPS genes was mapped using GSDS2.0.

A

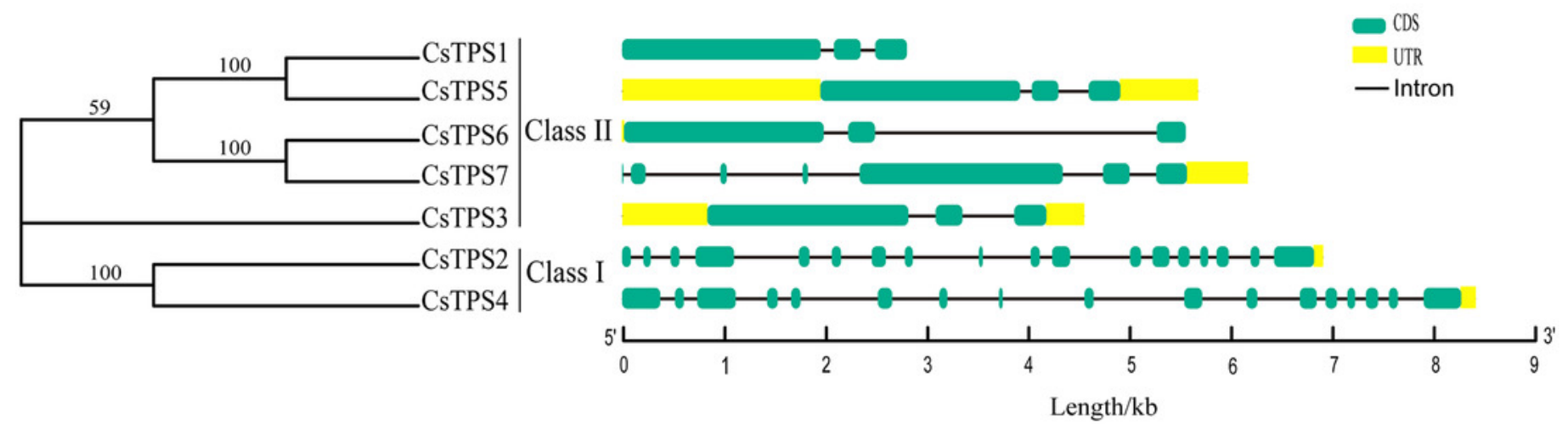




\section{Figure 2}

Evolutionary relationships of TPS family in various species.

A phylogenetic tree containing seven cucumber, 11 rice (OS), 11 Arabidopsis (At), and 20 soybean (Gm) TPS proteins was constructed using the maximum likelihood method (Cheng et al., 2018). The seven subgroups are colored differently. The four differently-colored shapes represent TPS proteins from four species. The black circle, yellow rectangle, red triangle, and blue star represent cucumber, rice, Arabidopsis, and soybean TPS proteins, respectively. 


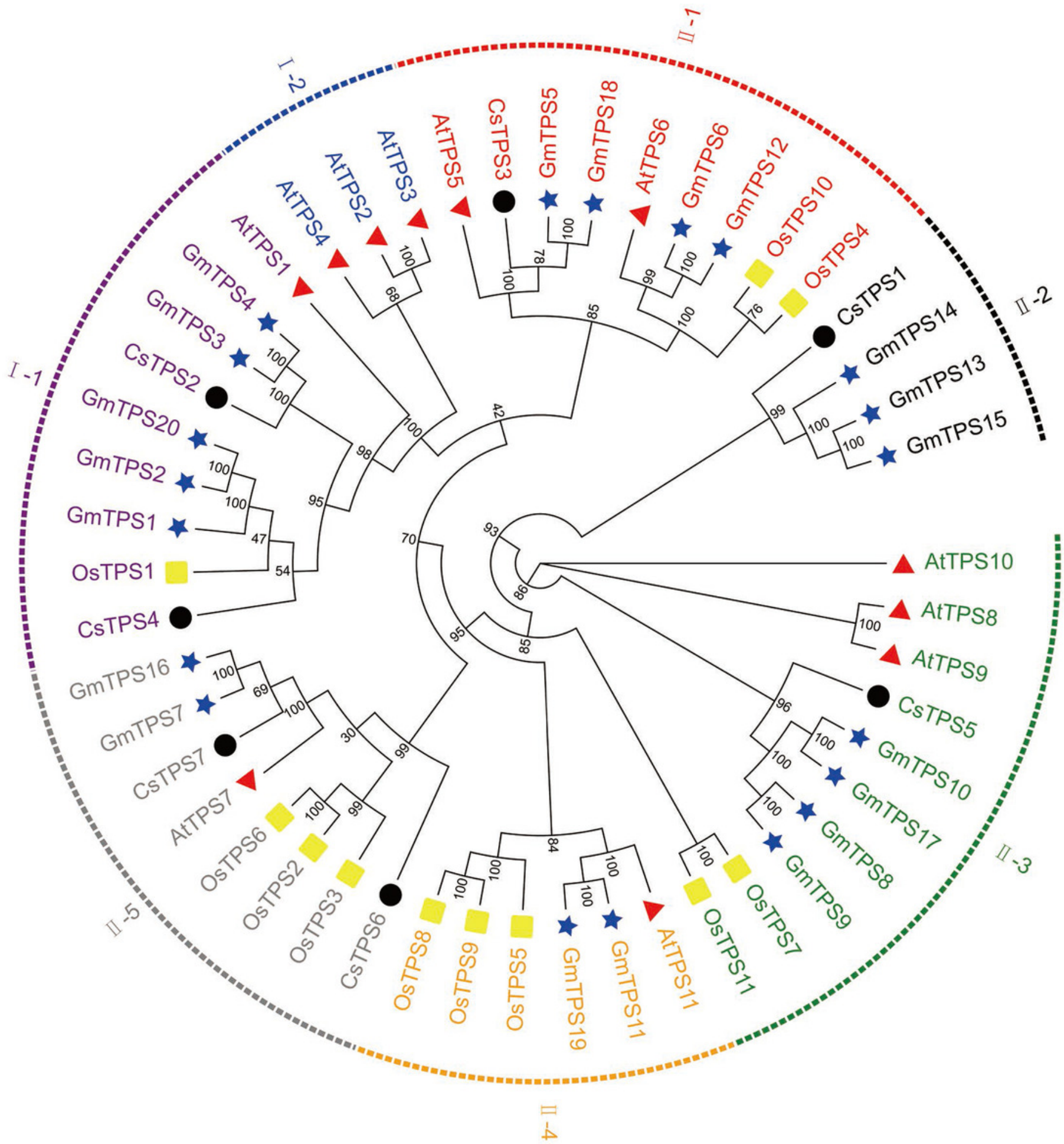


Figure 3

The motif composition and distribution of cucumber TPS proteins.

Colored boxes represent different conserved motifs.

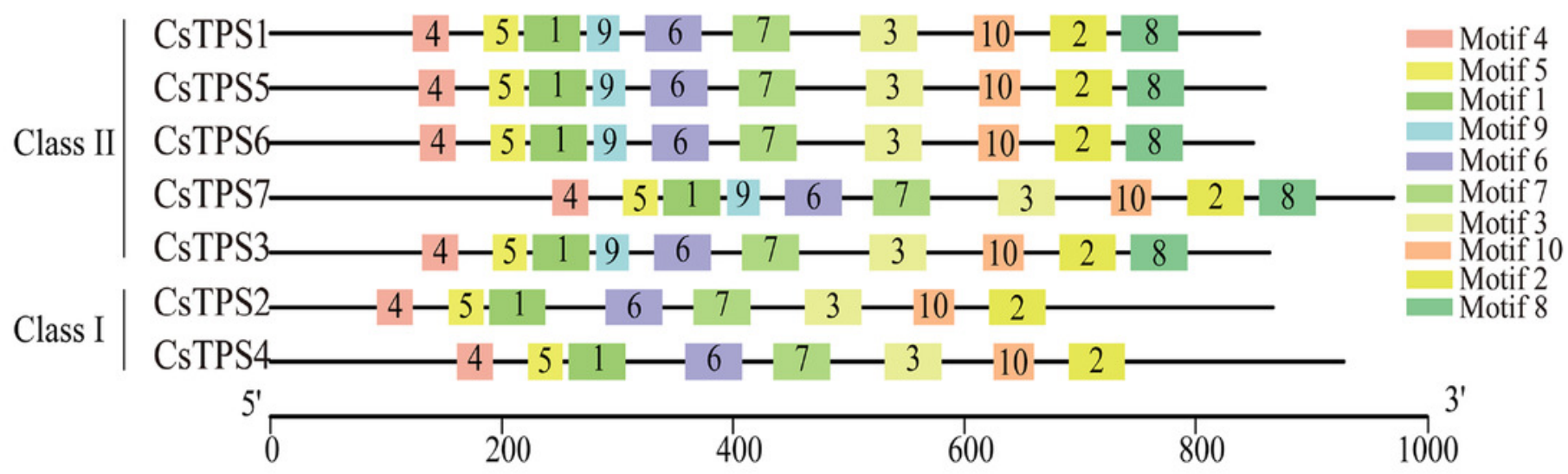


Figure 4

TPS gene locations in cucumber chromosomes.

The chromosomes are represented by green bars. .

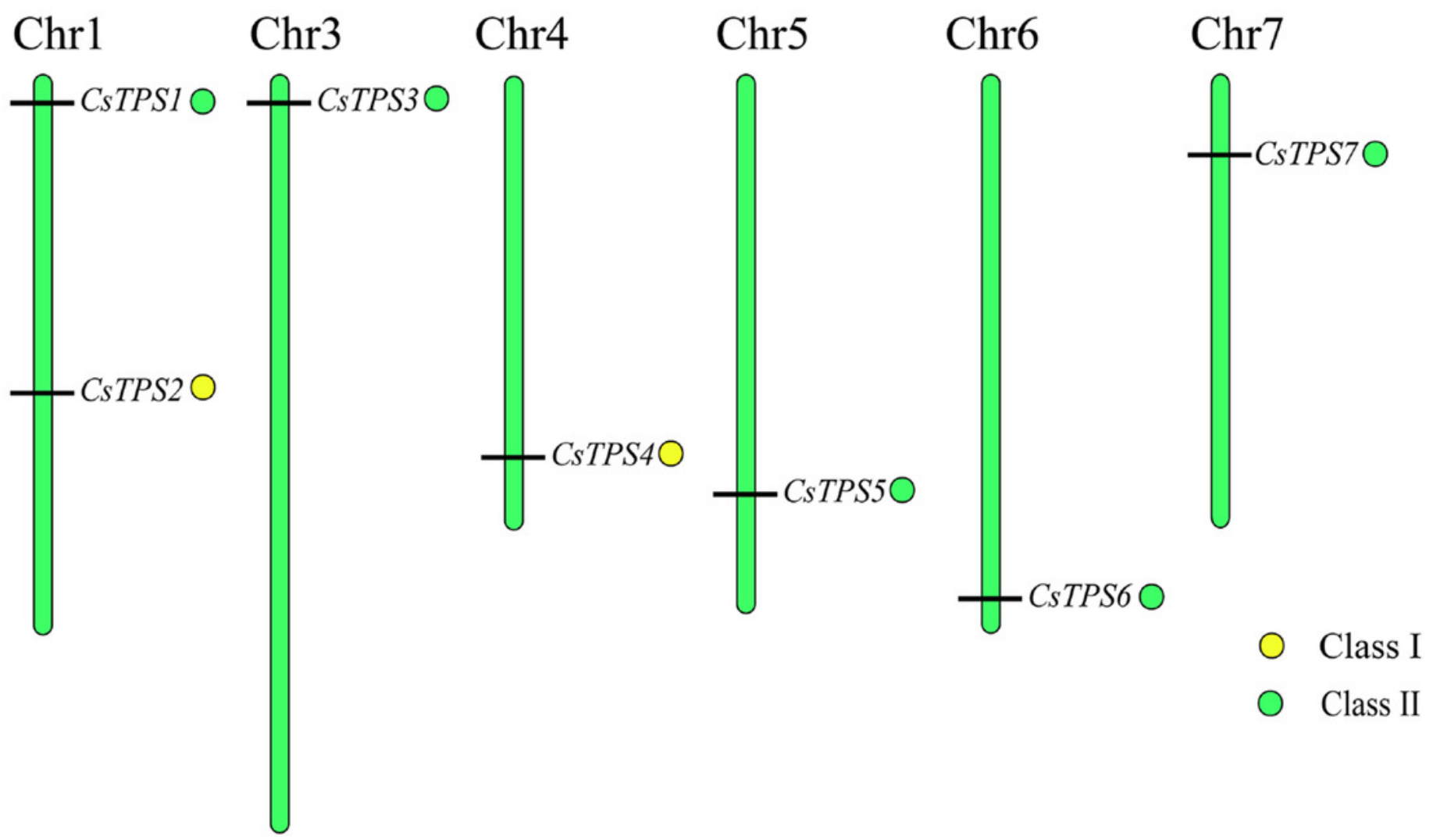


Figure 5

The distribution of cis-elements in cucumber TPS genes.

Colored rectangles represent different cis-acting elements.

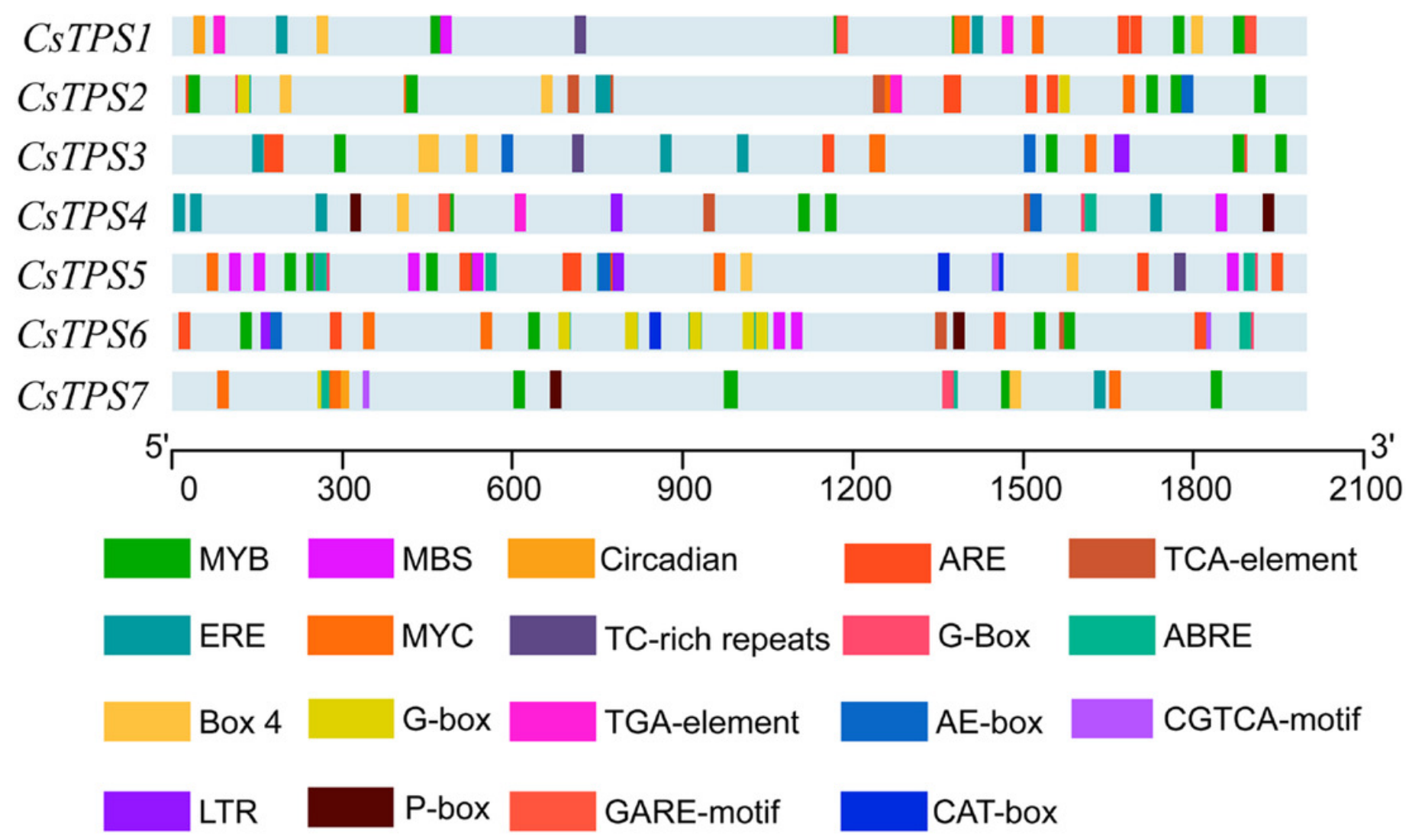


Figure 6

The number of Cis-acting elements in cucumber TPS genes. .

Stress-related elements

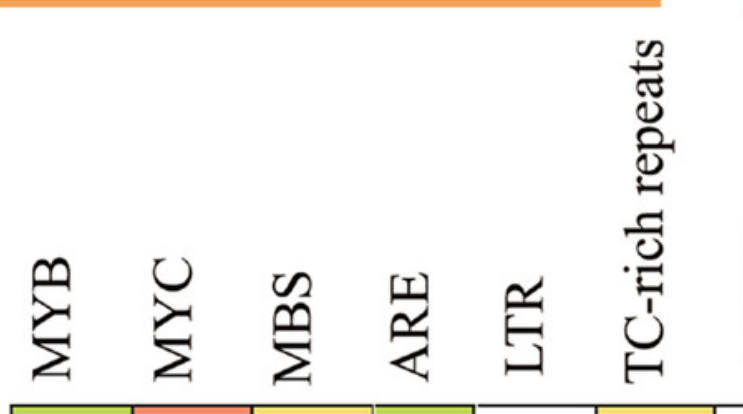

Phytohormone-responsive elements

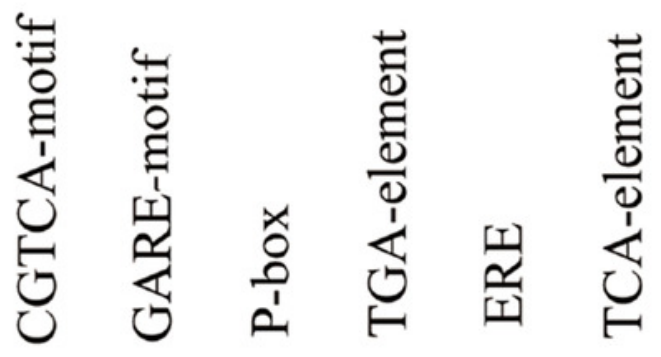

\section{CsTPS1 \\ CSTPS2 \\ CSTPS3 \\ CSTPS4 \\ CSTPS5 \\ CsTPS6 \\ CsTPS7}

\begin{tabular}{|l|l|l|}
\hline 6 & 3 & 1 \\
\hline 5 & 3 &
\end{tabular}

\begin{tabular}{|l|l|l|}
5 & 3 & \\
\hline 4 & 4 & \\
\hline
\end{tabular}

\begin{tabular}{|l|l|}
\hline 4 & 4 \\
\hline 3 &
\end{tabular}

3

\begin{tabular}{|l|l|l}
\hline 3 & & 1 \\
\hline 4 & 2 & 5
\end{tabular}

\begin{tabular}{|c|c|}
\hline 4 & 2 \\
\hline 5 & 3 \\
\hline
\end{tabular}

\begin{tabular}{l|l|l|l}
2 & & 1 & \\
\hline 5 & & &
\end{tabular}
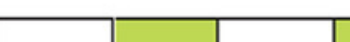

\begin{tabular}{|l|l|l|}
\hline 2 & 2 & 1 \\
\hline 1 & 2 & 3 \\
\hline & 3 & \\
\hline 1 & 4 & 2 \\
\hline & 1 & \\
\hline & & 2 \\
\hline & 1 & \\
\hline
\end{tabular}


Figure 7

Expression levels of CSTPS genes in root, stem, and leaf.

The expression patterns of CSTPS1-CSTPS7 in different tissues are shown in A-G, respectively. Error bars represent the standard error of three replicates. The relative expression of each gene in different tissues is expressed as mean $\pm S E(n=3)$. Bars with different lowercase letters were significantly different by Duncan's multiple range tests $(p<0.05)$.

A $\quad$ B

B $\quad$ C

C D

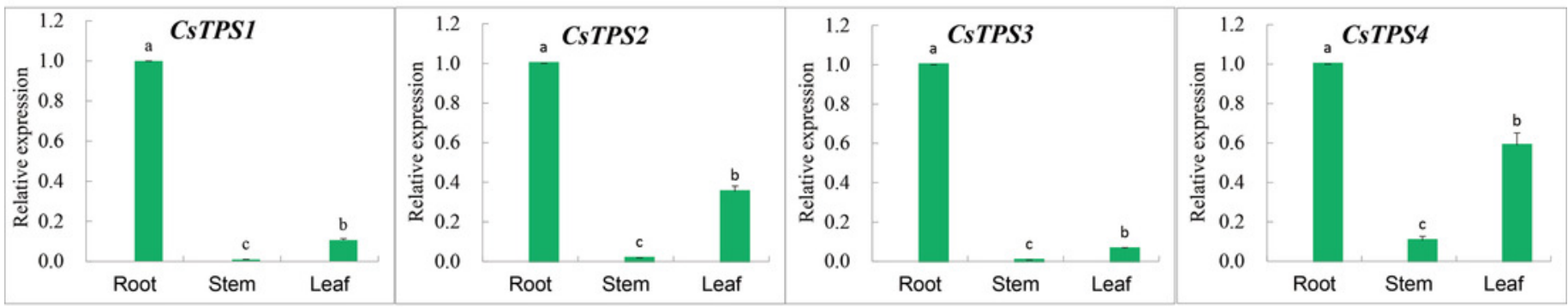

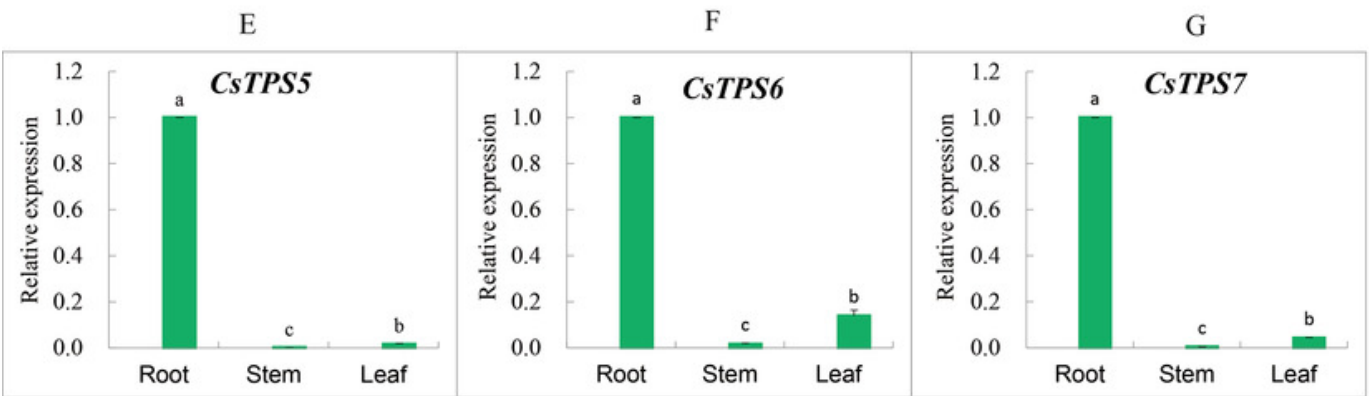




\section{Figure 8}

Expression levels of CSTPS genes under PEG, $\mathrm{NaCl}, \mathrm{H}_{2} \mathrm{O}_{2}, I A A$, mannitol, and sucrose treatments.

Seedlings were treated with $8 \%$ (w/v) PEG, $50 \mathrm{mM} \mathrm{NaCl}, 1 \mu \mathrm{M} \mathrm{IAA}, 8 \%$ (w/v) $\mathrm{H}_{2} \mathrm{O}_{2}, 50 \mathrm{mM}$ sucrose, and $50 \mathrm{mM}$ mannitol. The color scale represents fold changes normalized by log2 transformed data. Red represents up- regulated genes and blue represents down-regulated genes.

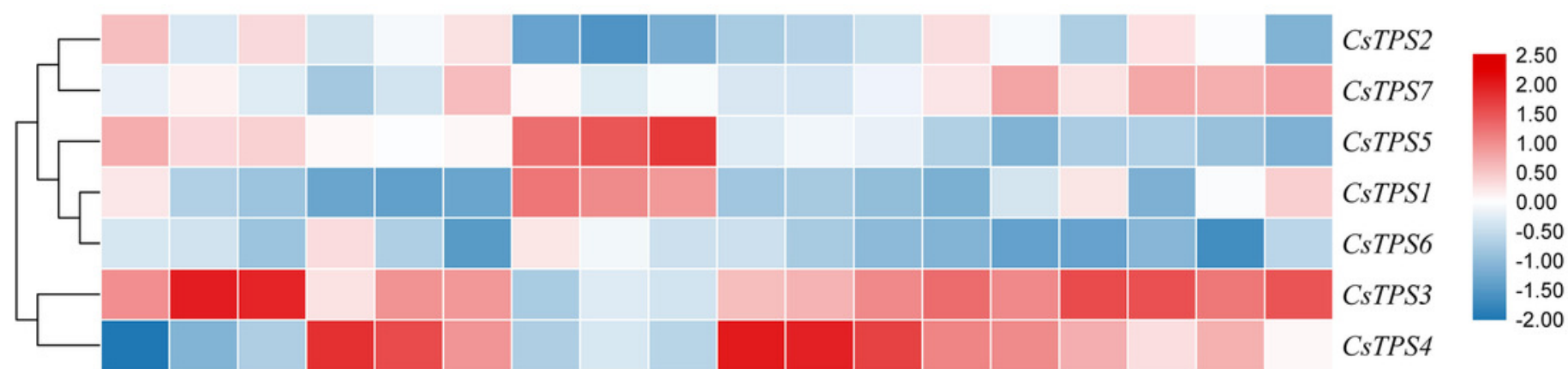


Table $\mathbf{1}$ (on next page)

Primer sequence for $q R T-P C R$ 


\begin{tabular}{lll}
\hline Gene name & Primer sequence $\left(5\right.$ ' to $^{3}$ ') & \\
\hline CsTPS1 & F: AAGTGGTGCTGTCAGGGTAAATCC & R: GCCCAGTAAGCAACATCGTGAGAG \\
CsTPS2 & F: AGCGTTGGTGGTTAGTCAGTGC & R: TGCTTTCTCTAGGGCTCTCTGTCC \\
CsTPS3 & F: TGGGCTCGGAGAAGATGTGGAAG & R: GTCGGGACGCACTTGAATCGG \\
CsTPS4 & F: ACCCTTCCATCCCGATCAGAGC & R: TCCTTGGTCCTCAACTCCTTCTGG \\
CsTPS5 & F: AAGCCAAGGAATTGCTGGACCATC & R: TGCGACCAACCCTTTGCTTACTC \\
CsTPS6 & F: CTGTCATGCCGCAAACTTCAATCG & R: AAACTTTCACGCCCTCTTCCACTG \\
CsTPS7 & F: AGACGGTGTTGCTTGGTGTTGATG & R: ACAGCCTTCCCTTGCCACTTTG \\
CsActin & F: TGGACTCTGGTGATGGTGTTA & R: CAATGAGGGATGGCTGGAAAA \\
\hline
\end{tabular}


Table 2 (on next page)

Fundamental information of CsTPS genes 


\begin{tabular}{|c|c|c|c|c|c|c|c|c|c|}
\hline Gene & Gene ID & $\begin{array}{l}\text { Gene } \\
\text { locus }\end{array}$ & $\begin{array}{l}\text { ORF } \\
\text { (bp) }\end{array}$ & $\begin{array}{l}\text { Amino } \\
\text { acid }\end{array}$ & $\begin{array}{l}\text { Molecular } \\
\text { weight/KDa }\end{array}$ & $\mathrm{pI}$ & $\begin{array}{l}\text { TPS domain } \\
\text { location }\end{array}$ & $\begin{array}{l}\text { TPP domain } \\
\text { location }\end{array}$ & $\begin{array}{l}\text { Subcellular } \\
\text { Localization }\end{array}$ \\
\hline CsTPS1 & Csa_1G005560 & Chr1 & 2568 & 855 & 97.14 & 6.34 & $53-540$ & $589-824$ & Chloroplast. Vacuole. \\
\hline CsTPS2 & Csa_1G467060 & Chr1 & 2604 & 867 & 97.87 & 7.02 & $21-492$ & $537-765$ & Chloroplast. Vacuole. \\
\hline CsTPS3 & Csa_3G009420 & Chr3 & 2595 & 864 & 97.46 & 5.73 & $62-548$ & $597-830$ & Cytoplasm. Vacuole. \\
\hline CsTPS4 & Csa_4G622880 & Chr4 & 2787 & 928 & 105.15 & 6.24 & $94-561$ & $606-834$ & $\begin{array}{c}\text { Cytoplasm. Vacuole. } \\
\text { Chloroplast. }\end{array}$ \\
\hline CsTPS5 & Csa_5G602180 & Chr5 & 2583 & 860 & 97.23 & 6.25 & $58-545$ & $594-829$ & Cytoplasm. Vacuole. \\
\hline CsTPS6 & Csa_6G520240 & Chr6 & 2553 & 850 & 96.31 & 5.50 & $59-544$ & $593-828$ & Cytoplasm. Vacuole. \\
\hline CsTPS7 & Csa_7G049190 & Chr7 & 2916 & 971 & 110.33 & 6.85 & $174-659$ & $708-943$ & Chloroplast. Vacuole. \\
\hline
\end{tabular}


Table 3 (on next page)

The secondary structures of CsTPS proteins 


\begin{tabular}{ccccc}
\hline Protein & Alpha helix (\%) & Beta turn (\%) & Random coil (\%) & Extended strand (\%) \\
\hline CsTPS1 & 42.81 & 4.21 & 35.79 & 17.19 \\
CsTPS2 & 42.68 & 5.19 & 35.87 & 16.26 \\
CsTPS3 & 43.63 & 5.44 & 34.26 & 16.67 \\
CsTPS4 & 43.97 & 5.39 & 37.07 & 13.58 \\
CsTPS5 & 41.98 & 5.35 & 35.23 & 17.44 \\
CsTPS6 & 43.06 & 5.18 & 34.82 & 16.94 \\
CsTPS7 & 39.03 & 5.46 & 36.05 & 19.46 \\
\hline
\end{tabular}


Table 4 (on next page)

Details of the 10 conserved motifs of cucumber TPS proteins 
1

\begin{tabular}{lclc}
\hline Motif & Width(aa) & Motif Sequence & Annotation \\
\hline Motif 1 & 50 & GFFLHSPFPSEIYRTLPVRDEJLRALLNADLIGFHTFDYARHFLSCCSR & Glyco-transf-20 \\
Motif 2 & 50 & WIQIAEPVMKLYTEATDGSHIETKESALVWHYQDADPDFGSCQAKELLDH & Trehalose_PPase \\
Motif 3 & 50 & KQLRHEKHYRYVSTHDVAYWSRSFLQDLERACRDHYRRRCWGIGFGLGFR & - \\
Motif 4 & 32 & FKCIPTFLPPEJLKQFYHGFCKQHLWPLFHYM & Glyco-transf-20 \\
Motif 5 & 31 & VVEVINPEDDYVWIHDYHLMVLPTFLRKRFN & Glyco-transf-20 \\
Motif 6 & 50 & FKGKKVJLGVDDLDIFKGINLKLLAFEQLLRQHPKWRGKAVLVQIANPAR & Glyco-transf-20 \\
Motif 7 & 50 & PGYEPIVLJDRPVPFHERIAYYAIAECCJVTAVRDGMNLVPYEYVVCRQG & Glyco-transf-20 \\
Motif 8 & 50 & KSGQHIVEVKPQGVSKGLVAEKILSSMAESGKLPDFVLCIGDDRSDEDMF & Trehalose_PPase \\
Motif 9 & 29 & YQSKRGYIGLEYYGRTVGIKILPVGIHMG & - \\
Motif 10 & 36 & EVISILNTLCDDPKNTVFIVSGRGRSSLGDWFGPCE & Trehalose_PPase \\
\hline
\end{tabular}

2 